\title{
THE EFFECT OF DIFFERENT SPATIAL WORKING MEMORY LOADS ON VISUAL SEARCH
}

\author{
Margit Höfler ${ }^{1,2}$, Sebastian A. Bauch², Elisabeth Englmair², Julia Friedmann-Eibler², \\ Corina Sturm ${ }^{2}$, \& Anja Ischebeck ${ }^{2}$ \\ ${ }^{1}$ Department for Clinical Neurosciences and Preventive Medicine, Danube University Krems (Austria) \\ ${ }^{2}$ Institute of Psychology, University of Graz (Austria)
}

\begin{abstract}
Working memory (WM) has been shown to be an important factor in visual search. For instance, there is evidence that both spatial and visual WM load lead to a decrease in search performance, resulting in a longer time to complete a search. However, the findings regarding search efficiency, i.e., search time as a function of display size, are less clear. This measure has been reported to be affected by spatial but not visual WM load. In three experiments, with approximately 20 participants each, we tested how two different types of spatial WM load affect visual search in terms of search performance and efficiency. In all experiments, participants were asked to memorize the spatial locations of two (low load) or four items (high load) presented either serially (Experiment 1) or simultaneously (Experiments 2 and 3). After that, they had to search for a target letter in a display of 5, 10 or 15 letters. In Experiment 3, participants additionally performed a verbal WM task. A control condition with no memory load (search only) was also included in each experiment. The results showed that, compared to the search-only condition, search times increased when spatial load was added. This was regardless of the type of spatial WM load. No search-time differences were found between the low and high-load condition. The additional verbal WM task had no effect on search performance. Furthermore, and in contrast to previous findings, search efficiency was not affected by either type of spatial WM load. These results suggest that visual search performance, but not search efficiency, is affected by spatial WM load.
\end{abstract}

Keywords: Visual attention, visual search, working memory load.

\section{Introduction}

Visual search is an everyday behavior in which we search for one or more target objects within a set of non-targets, so called distractors. In laboratory settings, visual search paradigms are often used to investigate attentional processes. Usually, participants are required to make a manual response regarding the absence or presence of a target in a search display that consists of a number of search items. The main variables of interest are commonly search performance (i.e., the response times), search accuracy (e.g., target hits and misses) and search efficiency (i.e., the search rate per additional item in the display). Theories of selective attention propose a distinction between parallel and serial visual search (e.g., Treisman \& Gelade, 1980, Treisman, 1988, Wolfe, 1994). In a parallel (feature or "pop-out") search, the target is distinct in one dimension from a set of rather homogeneous distractors (e.g., a blue ball among red balls) and can hence be found immediately and regardless of the number of objects in the search display. As a result, in parallel searches, response times are not affected by the presence or absence of the target. In a serial visual search, the search items are more heterogeneous and therefore have to be searched serially to determine whether the target is present or not. Consequently, serial target-absent searches last longer than target present searches because participants have to check all items in order to make a valid decision. In serial target-present searches, participants on average find the target when the display is searched halfway through (see e. g. Wolfe, 2020, for a review).

Previous research has indicated that working memory (WM) is an important factor in visual search (e.g., Höfler, Gilchrist, \& Körner, 2014, Oh \& Kim, 2004; Woodman \& Luck, 2004). In experiments which test the influence of WM on visual search, participants are typically presented with a set of objects and are asked to memorize the locations or specific features (e.g., the color) of these objects while performing a subsequent visual search task. Typically, findings show that overall search performance decreases in such dual-task paradigms, i.e., the search takes longer due to the memory load 
(e.g., He \& McCarley, 2010, Oh \& Kim, 2004; Woodman \& Luck, 2004). The findings regarding WM load on search efficiency (i.e., the search rate per item in the display), remain rather unclear. For instance, Woodman, Vogel and Luck (2001) showed that memorizing object features such as colors prior search has no effect on search efficiency, whereas memorizing the spatial location of objects affects both performance and efficiency (e.g., Oh \& Kim, 2004; Woodman \& Luck, 2004). These findings suggest that spatial rather than visual WM is involved during visual search. However, Anderson, Mannan, Rees, Sumner, and Kennard (2008) showed that verbal WM load affects search efficiency in serial searches to the same extent as spatial WM load. However, results of Solman, Cheyne and Smilek (2011) suggest that (non-spatial) WM load does not necessarily affect search efficiency. By analyzing eye movements of their participants while they were searching a display under different WM load conditions, they found that fixations were made farther away from search items (i.e., they were less precise) when non-spatial WM load was added, and previously inspected locations were more often revisited.

In Oh and Kim (2004), participants were required to memorize four item locations at once prior to the search task, whereas in Woodman and Luck (2004), they had to memorize two item locations presented serially prior to the search to prevent participants from forming a shape-based mental representation that would not require spatial WM resources. However, these different types of WM load (all at once vs. serially) could have actually affected search differently. Moreover, in both studies, participants were required to perform an articulatory suppression task throughout the experimental trial. It is unclear whether the verbal task might have even increased the effect of the visuo-spatial WM load. In the following experiments, we therefore wanted to test in greater detail whether and how different types visuo-spatial WM load affect a visual-search task that consists of letter stimuli. In all experiments, we had participants search for a target letter in a letter display with 5, 10, or 15 different letters while they were additionally asked to memorize the locations of 0, 2 or 4 squares. In Experiment 1, these squares were presented serially; in Experiments 2 and 3, they were presented at once. In Experiment 3, participants were additionally required to perform an articulatory suppression task. For all experiments, we expected a decrease in search performance when WM load is added such that the searches should last longer with increasing WM load. However, we expected that the effect of WM load on the search efficiency, as measured by the search rate, depends on the type and the amount of WM load. That is, increasing WM load should lead to less efficient searches, and this effect should be more pronounced in Experiment 3 (verbal and spatial WM load) than in Experiment 2 (spatial WM only). Furthermore, we expected that search efficiency to be more affected when the to-be-remembered locations were presented serially than all at once (Experiments 1 vs. Experiment 2).

\section{Method}

\subsection{Design}

In all three experiments, a 3 (memory condition) $\times 3$ (search condition) $\times 2$ (target presence) within-subjects design was used. That is, participants had to remember either 0,2 , or 4 item locations (no vs. low vs. high memory load) before searching a display consisting of either 5, 10 or 15 letters. Participants were asked to memorize the items presented prior search and to indicate the target's presence in the search task via button press. The target was absent on half of the trials. We measured manual response times from display onset to the manual response as the main dependent variable.

\subsection{Participants}

We recruited 20 participants in Experiment 1 (18 female; $M=23.3$ years; $S D=2.2), 20$ in Experiment 2 (16 female; $M=23.8$ years; $S D=3.9$ ) and 24 participants in Experiment 3 (12 female; $M=23.2$ years; $S D=2.3$ ). All reported normal or corrected-to-normal vision. All of them gave written informed consent before the start of the experiment and received course credit for their participation. The experiments were approved by the ethics committee of the University of Graz.

\subsection{Stimuli and procedure}

In all experiments, a fixation disc was presented at the center of the display for $750 \mathrm{~ms}$ at the beginning of a trial (see Figure 1). In Experiment 3 only, two different numbers, randomly selected from the numbers 1 to 9 , were then presented for $1,000 \mathrm{~ms}$ and the participants were asked to repeat these numbers aloud throughout the whole trial. Then the fixation disc was presented again for $750 \mathrm{~ms}$, followed by the memory display for 1,000 ms. Participant's task was to memorize the location of 0,2 or 4 squares $(0.9 \times 0.9$ degrees of visual angle; d.v.a. $)$ that were located randomly at 12 possible locations around the center of the display. 
In Experiment 1, the 2 or 4 memory items were presented serially for $500 \mathrm{~ms}$ and $250 \mathrm{~ms}$ respectively (i.e., $1000 \mathrm{~ms}$ in total). In Experiments 2 and 3, all memory items were presented at once for $1,000 \mathrm{~ms}$, followed by the search display. The target letter was announced via head set simultaneously with the onset of the search display. Participant's task was to search for the target in the display consisting of 5,10 or 15 letters, respectively and to give a present/absent response on a two-button response box. The letters were presented within an invisible $7 \times 7$ grid $(25.9 \times 25.9$ d.v.a. $)$ and were surrounded by a circle with a diameter of 0.9 d.v.a. After the manual response, a test display was presented and participants had to decide via a button press whether the position of the single memory item presented in this test display matched with one of the to-be-remembered positions from the memory display. In case of the no-load condition, the memory display and the test display remained blank. After this, the display was cleared, and a new trial started.

Participants sat in a darkened, sound-proof cabin at a distance of about $63 \mathrm{~cm}$ in front of a 21 ', CRT monitor with a resolution of $1,152 \times 864$ pixels and a refresh rate of $100 \mathrm{~Hz}$. A chin rest was used to minimize head movements. Stimuli were created using Microsoft Visual C++ 2008 Express Edition. Each participant completed three blocks of 90 trials each; the memory condition was varied block-wise whereas all other conditions were varied within blocks. The sequence of memory conditions was counterbalanced across participants. Before each block, 10 practice trials were conducted.

Figure 1. Sample Procedure of a trial in Experiment 3 (Stimuli are not drawn in scale).

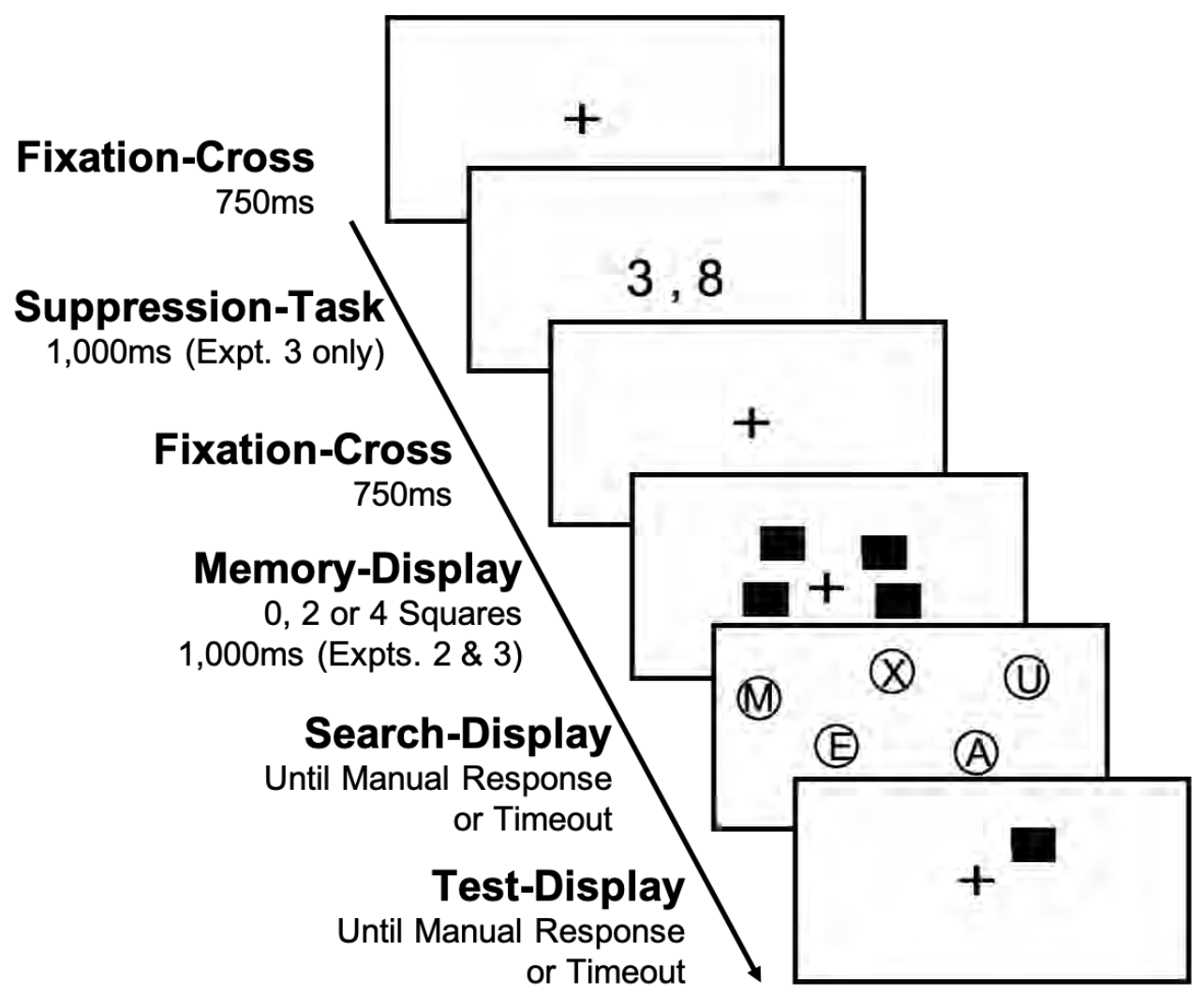

\section{Results}

\subsection{Error rates}

In all experiments, we excluded data from participants who had a higher error rate than $10 \%$ in the visual search task or when the memory task was conducted on chance level, as indicated by a binomial test. In Experiment 1, data of 17 participants entered the analysis. The error rate in the search task was $M=3.7 \%(S D=1.6 \%)$; the error rate in the memory task was $M=18.2 \%(S D=6.9 \%$, low load) and $M=28.6 \%(S D=5.9 \%$, high load $)$. A paired $t$-test showed that the error rate was significantly higher for the high-load vs. the low-load condition, $t(16)=6.35, p<.001$.

In Experiment 2, data from 14 participants were included in the analysis. The error rate in the search task was $M=3.4 \%(S D=2.5 \%)$; the error rate in the memory task was $M=14.5 \%(S D=7.2 \%$ low load) and $M=26.0 \%(S D=9.1 \%$, high load $)$. This latter difference was reliable, $t(13)=7.89$, 
$p<.001$. In Experiment 3, data of four participants had to be excluded from analysis because of the criteria defined above. For the 20 remaining participants, the average error rate for the search task was $M$ $=2.9 \%(S D=2.3 \%)$ and for the memory task $M=16.3 \%(S D=8.0 \%$, low load $)$ and $M=23.2 \%(S D$ $=8.1 \%$, high load). A $t$-test for repeated measures indicated again that the error rate for the high-memory load condition was significantly higher than for the low-load condition, $t(19)=3.87, p=.001$.

\subsection{Search performance}

Table 1 shows the mean response times and standard deviations for all load conditions and display sizes averaged across participants' individual means for all three experiments. A $3 \times 3 \times 3$ ANOVA for repeated measures with display size $(5,10$, or 15 letters) and memory condition $(0,2$, or 4 memory items) as within-subjects factors and experiment (1 to 3 ) as between-subjects factor showed no effect of experiment, $F<1$, but a significant effect of display size, $F(1.25,59.96)=1134.94, p<.001, \eta_{p}{ }^{2}$ $=.96$. Bonferroni-Holm corrected $t$-tests indicated that participants needed longer to find the target as display size increased (all $p \mathrm{~s}<.001$ ), reflecting a standard finding in serial visual search (e.g, Wolfe, 2020). Furthermore, the main effect of load condition was also significant, $F(2,96)=28.75, p<.001, \eta_{p}{ }^{2}$ $=.37$, such that response times increased from load condition 0 to load condition $2(p<.001)$ while no such difference was found for load conditions 2 vs $4(p=.35)$. However, none of the interactions were significant (all $p s>.20)$.

Table 1. Mean response times and search rates (standard deviation) for all experiments and conditions.

\begin{tabular}{lcccc} 
& & No load & Low load & High load \\
\hline Expt. 1 & DS 5 & $1,901(228)$ & $2,168(463)$ & $2,265(637)$ \\
& DS 10 & $3,031(353)$ & $3,411(644)$ & $3,433(773)$ \\
Expt. 2 & DS 15 & $4,040(658)$ & $4,274(816)$ & $4,291(950)$ \\
& Search rate / item & $214(49)$ & $211(42)$ & $203(52)$ \\
& DS 5 & $1,829(242)$ & $2,217(448)$ & $2,174(1,012)$ \\
& DS 10 & $2,931(403)$ & $3,478(763)$ & $3,381(696)$ \\
& DS 15 & $4,061(685)$ & $4,504(1012)$ & $4,582(831)$ \\
& Search rate / item & $220(57)$ & $219(73)$ & $232(55)$ \\
& DS 5 & $1,931(250)$ & $2,173(385)$ & $2,368(507)$ \\
& DS 10 & $3,139(445)$ & $3,497(586)$ & $3,672(745)$ \\
& DS 15 & $4,205(589)$ & $4,584(845)$ & $4,700(795)$ \\
& Search rate / item & $227(42)$ & $241(56)$ & $233(48)$ \\
\hline
\end{tabular}

Note. DS = Display size.

\subsection{Search efficiency}

The average search rates, indicated by the search time per item as a function of display size for each experiment, can be found in Table 1. A mixed-way ANOVA with WM load condition as within-subject and experiment as between-subject factor showed that there was neither a reliable difference across experiments, $F(2,48)=1.48, p=.24$ nor WM load conditions, $F<1$. Also, the interaction was not significant, $F<1$. This suggests that the WM load used in the experiments does not affect search efficiency.

\section{Conclusion}

The aim of the current experiment was to investigate whether and how different types of visuo-spatial working memory load affect a visual search task. In line with previous findings (e.g., Oh \& Kim, 2004, Woodmann \& Luck, 2004), we showed that search performance decreased with increasing spatial memory load. That is, if participants had to memorize two item locations prior to visual search, search times increased in the visual search task compared to a control condition without WM load. However, there was no additional increase in search time from the two-item to the four-item WM load condition. These results were observed regardless of whether the locations to be remembered were 
presented simultaneously or serially, or whether participants had to perform an additional verbal suppression task. This suggests that these types of spatial WM load affect visual search in a similar way. The lack of further increase from the two- to the four-item WM condition indicates that a two-item load appears to occupy all WM resources.

Furthermore, although both visuo-spatial tasks affected search performance significantly, we could not replicate the findings from Oh and Kim (2004) and Woodman and Luck (2004) that increasing spatial WM load reduced search efficiency as well. Search did not become more inefficient when spatial WM load was added. Findings in which search efficiency is not affected by WM load are commonly observed in experiments that use non-spatial WM tasks (e.g., Solman, et al., 2011, Woodman \& Luck, 2001). It is often argued that in such a case the search process is not affected by the WM load. However, Solman et al. suggested that (non-spatial) WM load does not necessarily affect search efficiency. They monitored participants' eye movement behavior and investigated the time spent in three different phases of the search: between the onset of the display until the first saccade, between the first saccade and fixation of the target, and between fixation of the target and the manual response. Findings showed that WM load affected all phases of the search and that fixations tended to become imprecise. In the light of the current findings, it is therefore possible that eye movement behavior also changed during search when visuo-spatial WM load was increased, although this change is not reflected in the analysis of search efficiency. Hence, additional experiments in which the eye movements are monitored during search are necessary to further investigate these diverging effects of WM load on search performance and search efficiency.

\section{Acknowledgements}

This work was supported by the Austrian Science Fund (FWF): P28546 and P33074. We thank Fernanda Hoffmann for her support during the preparation of this document.

\section{References}

Anderson, E. J., Mannan, S. K., Rees, G., Sumner, P., \& Kennard, C. (2008). A Role for Spatial and Nonspatial Working Memory Processes in Visual Search. Experimental Psychology, 55(5), 301-312. doi:10.1027/1618-3169.55.5.301

He, J., \& McCarley, J.S. (2010). Executive working memory load does not compromise perceptual processing during visual search: Evidence from additive factors analysis. Attention, Perception, \& Psychophysics, 72(2), 308-316. doi:10.3758/APP.72.2.308

Höfler, M., Gilchrist, I. D., \& Körner, C. (2014). Searching the same display twice: Properties of short-term memory in repeated search. Attention, Perception, \& Psychophysics, 76(2), 335-352. doi: $10.3758 / \mathrm{s} 13414-013-0589-8$

Oh, S. H., \& Kim, M. S. (2004). The role of spatial working memory in visual search efficiency. Psychonomic Bulletin \& Review, 11(2), 275-281. doi:10.3758/BF03196570

Solman, G. J. F., Cheyne, J. A., \& Smilek, D. (2011). Memory load affects visual search processes without influencing search efficiency. Vision Research, 51(10),1185-1191. doi:10.1016/j.visres.2011.03.009

Treisman, A. (1988). Features and objects: The fourteenth Bartlett memorial lecture. The Quarterly Journal of Experimental Psychology, 40(2), 201-237. doi: 10.1080/02724988843000104

Treisman, A. M., \& Gelade, G. (1980). A Feature-Integration Theory of Attention. Cognitive Psychology, 12(1), 97-136. doi:10.1016/0010-0285(80)90005-5

Wolfe, J. M. (1994). Guided search 2.0 A revised model of visual search. Psychonomic Bulletin \& Review, 1(2), 202-238. doi: 10.3758/BF03200774

Wolfe, J. M. (2020). Visual search: How do we find what we are looking for?. Annual review of vision science, 6, 539-562. doi:10.1146/annurev-vision-091718-015048

Woodman, G. F., \& Luck, S. J. (2004). Visual search is slowed when visuospatial working memory is occupied. Psychonomic Bulletin and Review, 11(2), 269-274. doi:10.3758/BF03196569

Woodman, G. F., Vogel, E. K., \& Luck, S. J. (2001). Visual Search Remains Efficient when Visual Working Memory is Full. Psychological Science, 12(3), 219-224. doi:10.1111/1467-9280.00339 